\title{
The current status of acellular pertussis vaccines
}

The position with regard to acellular pertussis vaccines has been reviewed at meetings held at the Istituto Superiore di Sanita, Italy in 1995 [1], the National Institutes of Health, USA [2] and the National Institute for Biological Standards and Control, UK in 1996 [3]. These meetings reviewed the evidence for efficacy of vaccines that emerged from clinical trials performed in Germany, Italy, Senegal and Sweden over the last few years. They also considered the methodology currently available for laboratory evaluation of these vaccines and particularly for assuring consistency of postclinical trial production lots. The data presented indicated that there are still many unresolved problems relating to the introduction of acellular pertussis vaccines into routine immunisation programmes.

In the first instance, the evidence in support of clinical efficacy is far from straightforward. The composition of the vaccines varied widely between manufacturers, ranging from monovalent pertussis toxoid vaccine, through bivalent (pertussis toxoid + filamentous haemagglutinin), trivalent (pertussis toxoid + filamentous haemagglutinin + pertactin), pentavalent (pertussis toxoid + filamentous haemagglutinin + pertactin + fimbrial agglutinogens (fims) 2 and 3 ) to the copurified Takeda type vaccine (predominantly filamentous haemagglutinin with pertussis toxoid, pertactin, fim 2 and many minor components). Even for apparently similar formulations the method of detoxification of the pertussis toxin differed. Most manufacturers used chemical treatment, usually formaldehyde or glutaraldehyde or both, but hydrogen peroxide or genetic detoxification, respectively, were used for two preparations $[4,5]$. Furthermore, the quantity of individual components differed by as much as 10fold between apparently similar formulations. In addition, the specific case definitions for pertussis, the immunisation schedules and study designs varied widely between trials. Whole-cell pertussis vaccine of various origins (French, German, North American and British) were included in some but not all studies. These factors make a valid direct comparison of the results of these studies almost impossible.

At first sight, several of the vaccines - the two trivalent and the pentavalent preparation - appear to have demonstrated almost identical efficacy, with point estimates of c. 84\% [6]. However, detailed examination of the durability of the protection suggests a rather better performance for the genetically detoxified trivalent vaccine than for the others. Comparison of serological responses to the antigens represented in the vaccines also showed considerable variation [7].

Examination of the cell-mediated responses of some of the vaccine recipients was performed in the Stockholm, Italian and German studies. These showed that some but not all recipients produced Thl or Th0 type responses to Bordetella pertussis antigen $[1,8]$. The results were less clear-cut than for experimental studies in mice which have indicated a definite role for Th1 responses in protection against respiratory challenge with $B$. pertussis [9].

All of the vaccines except the monovalent preparation were compared against whole-cell vaccines. One of the latter, the Connaught vaccine, gave very poor protection in the Stockholm [10] and Italian [11] trials, with efficacy values of approximately $36-48 \%$. In contrast, the Behringwerke, Evans-Wellcome and Merieux whole-cell vaccines gave efficacies of at least $95 \%$ in the German, Stockholm and Senegal trials respectively. All four vaccines had passed the intracerebral challenge potency assay, although the Connaught preparation had been assayed according to the US criteria which allow a less stringent interpretation than those used in Europe. As these results allow direct comparison of all types of vaccine used in the same trials, they show that the most effective wholecell preparations are still significantly more protective than even the best acellular formulations, although the $\mathrm{S}$ component vaccine gave comparable efficacy in the Stockholm trial [12].

This raises a question for those responsible for implementation of vaccination policy. Should they opt for acellular vaccines of low reactogenicity, or more reactogenic whole-cell vaccines of greater efficacy? The evidence on reactogenicity is limited to relatively small sample populations and the type of study used in the various trials can give no information on rare events such as encephalopathy. Although, in principle, the acellular vaccines seem to offer more defined preparations free of potentially toxic materials, the methods available for assuring their consistency have severe limitations. Thus, no true potency test has been used on clinical trial lots. Manufacturers have relied on immunogenicity assays which measure total rather than functional antibody and do not take account of cell-mediated responses. 
This means that they may be unable to ensure that subsequent production lots reproduce the protective properties of trial lots. Alternative methods are under investigation, including use of respiratory challenge models, with either intranasal or aerosol inoculation. A modified intracerebral challenge system is used in Japan, apparently with success [13]. There is understandable reluctance to adopt this procedure in Western countries given the experience of a similar method in controlling whole-cell vaccines. It is clear that there is a pressing need to develop new control procedures for acellular vaccines before their use becomes widespread. If this is not done, the situation encountered in the early days of whole-cell vaccines could arise again, with serious implications for immunisation programmes.

\section{J. CORBEL and D. K. L. XING} Division of Bacteriology, National Institute for Biological Standards and Control, Potters Bar, EN6 3QG

\section{References}

1. Brown F, Greco D, Mastrantonio P, Salmaro S, Wassilak S. Pertussis Vaccines Trials, Rome. 30 Oct.-1 Nov. 1995: Trial synopses Rome: Istituto Superiore di Sanita, 1995. Dev Biol Stand 1997; 89 (in press).

2. Pertussis Conference, National Institutes of Health, Acellular Pertussis Vaccine Trials: results and impact on U.S. Public
Health. 3-5 June 1996, National Institutes of Health, Washington, DC, USA.

3. WHO/IABS/NIBSC International Meeting on the Control and Standardisation of Acellular Pertussis Vaccines, 26-27 Sept. Potters Bar, UK. Dev Biol Stand 1997; 89: (in press).

4. Ibsen PH. The effect of formaldehyde, hydrogen peroxide and genetic detoxification of pertussis toxin on epitope recognition by murine monoclonal antibodies. Vaccine 1996; 14: 359-368.

5. Poland GA. Acellular pertussis vaccines: new vaccines for an old disease. Lancet 1996; 347: 209-210.

6. Schmitt HJ. Results of a household contact protective efficacy study. Presented at: Caring for the new generation, Conference Sorrento, Italy, 6-7 April, 1995. Abstract (p. 37).

7. Edwards KM, Meade BD, Decker MD et al. Comparison of 13 acellular pertussis vaccines: overview and serologic response. Pediatrics 1995; 96: 548-557.

8. Zepp F, Knuf M, Habermehl $\mathrm{P}$ et al. Pertussis-specific cellmediated immunity in infants after vaccination with a tricomponent acellular pertussis vaccine. Infect Immun 1996; 64: $4078-4084$

9. Barnard A, Mahon BP, Watkins J, Redhead K and Mills KHG. Th1/Th2 cell dichotomy in acquired immunity to Bordetella pertussis: variables in the in vivo priming and in vitro cytokine detection techniques affect the classification of T-cell subsets as Th1, Th2 or Th0. Immunology 1996; 87: 372-380.

10. Gustafsson L, Hallander HO, Olin P, Reizenstein E, Storsaeter J. A controlled trial of a two-component acellular, a fivecomponent acellular, and a whole-cell pertussis vaccine. $N$ Engl J Med 1996; 334: 349-355.

11. Greco D, Salmaso S, Mastrantonio P et al. A controlled trial of two acellular vaccines and one whole-cell vaccine against pertussis. N Engl J Med 1996; 334: 341-348.

12. Olin P, Gustafson L, Rasmussen F, Hallonder H, Heijbel H Gottfarb P. Efficacy trial of acellular pertussis vaccines. Technical Report II. 1997. Stockholm, Swedish Institute for Infectious Disease Control.

13. Sato Y, Sato H. Animal models in pertussis. In: Wardlaw AC, Parton $R$ (eds) Pathogenesis and immunity in pertussis Chichester, John Wiley and Sons. 1988: 309-325. 